\title{
Expression of Caveolin-1 in Rat Urinary Bladder with Cyclophosphamide-Induced Cystitis
}

\author{
Sun-Ouck Kim, Seung Hee Song, Seung-Chul Lee ${ }^{1}$, Kyung A Cho ${ }^{2}$, Ho Song Yu, In Sang Hwang, Eu Chang Hwang, \\ Dongdeuk Kwon \\ Departments of Urology, ${ }^{1}$ Dermatology, and ${ }^{2}$ Biochemistry, Chonnam National University Hospital, Chonnam National University Medical School, Gwangju, \\ Korea
}

\begin{abstract}
Purpose: The purposes of this study were to investigate the effect of cyclophosphamide (CYP)-induced inflammatory cystitis on caveolin 1 in rat urinary bladder and to determine the role of these molecules in the bladder dysfunction that occurs in inflammatory change in rat urinary bladder.

Methods: Female Sprague-Dawley rats were divided into control $(n=30)$ and experimental $(n=30)$ groups. Cystitis in experimental group was induced by intraperitoneal injection of CYP $(200 \mathrm{mg} / \mathrm{kg})$. The control group underwent an intraperitoneal saline injection. After 3 days, urodynamic studies were done to measure the contraction interval and contraction pressure. The expression and cellular localization of caveolin 1 were determined by Western blot and immunofluorescent study in rat urinary bladder.

Results: In cystometrograms, the contraction interval (minute) was significantly increased in the CYP-induced cystitis rats $(15.8 \pm 1.5)$ than in the control group $(6.3 \pm 0.5)(\mathrm{P}<0.05)$. Conversely, the average contraction pressure $(\mathrm{mmHg})$ was significantly higher in the CYP-induced cystitis rats $(15.6 \pm 1.7)$ than in the control group $(11.3 \pm 0.5)(\mathrm{P}<0.05)$. Caveolin 1 was expressed in the capillaries, arteriols and venules. The protein expression of caveolin 1 was significantly decreased in the CYP-induced cystitis rats $(\mathrm{P}<0.05)$.

Conclusions: Inflammatory change of urinary bladder maybe causes a significant change in the expression of caveolin 1 . These findings suggest that caveolin 1 might have a functional role in the bladder dysfunction related with cystitis in rat urinary bladder.
\end{abstract}

Keywords: Caveolin 1; Cystitis; Rats

\section{INTRODUCTION}

Traditionally, the urothelium was considered to be a simple passive barrier between the urinary tract and urine. Recently, however, the urothelium is now understood to be a responsive organ capable of sensing a variety of signals from the urinary bladder [1].

Caveolae are flask shaped plasma membrane invaginations that are thought to play a role in important cellular processes of cell surface signaling [2]. Caveolins are major constituents of caveolae and caveolins are required for the formation of caveo- lae [3]. The caveolae family proteins consist of 3 isoforms, caveolin (caveolin 1, 2, 3). Among them, caveolin 1 is the best characterized isoform and shown to play roles in receptor mediated signal transduction pathway regulation. Functional roles of caveolin-1 in the bladder are beginning to emerge with the study of genetic deletion of caveoln-1 in animal study. Caveolin 1 knockout mice in the bladder exhibit several urological disorders including decrease detrusor contractility on stimulation with carbachol $[4,5]$. Our group has previously reported that the effect of hormonal alteration on caveoln 1 in urinary bladder and determine the role of this molecule in the detrusor
Corresponding author: Sun-Ouck Kim

Department of Urology, Chonnam National University Hospital, Chonnam National University Medical School, 42 Jebong-ro, Dong-gu, Gwangju 501-757, Korea

Tel: +82-62-220-6705 / Fax: +82-62-227-1643 / E-mail: seinsena@hanmail.net Submitted: December 13, 2012 / Accepted after revision: December 20, 2012
This is an Open Access article distributed under the terms of the Creative Commons Attribution Non-Commercial License (http://creativecommons.org/licenses/by-nc/3.0/) which permits unrestricted non-commercial use, distribution, and reproduction in any medium, provided the original work is properly cited. 
overactivity that occurs in hormonal alteration [6]. We found that hormomal alteration causes a significant change in bladder contraction and the expression of caveolin 1 in ovariectomized rat.

Most studies to date have demonstrated the caveolin expression in detrusor muscle of urinary bladder. However, there have been no studies to investigate the combined changes in expression of caveolin 1 and functional activity of this protein in response to clinically important cystitis condition. The purposes of this study were to investigate the effect of cyclophosphamide (CYP)-induced inflammatory cystitis on caveolin 1 in rat urinary bladder and to determine the role of this molecule in the bladder dysfunction that occurs in associated with inflammatory change in rat urinary bladder.

\section{MATERIALS AND METHODS}

\section{Experimental Model}

Female Sprague-Dawley rats were divided into control $(\mathrm{n}=30)$ and experimental $(\mathrm{n}=30)$ groups. Cystitis in experimental group was induced by intraperitoneal injection of CYP $(200 \mathrm{mg} / \mathrm{kg})$ [7]. The control group underwent an intraperitoneal saline injection. After 3 days, urodynamic studies were done to measure the contraction interval and contraction pressure. All experimental animals were fed a standard diet up until the day before the experiment. Animals with an estrous cycle confirmed via a vaginal smear were premedicated with xylazine $(2.2 \mathrm{mg} / \mathrm{kg}$, intramuscular [IM]) and anesthetized with a zolazepam/tiletamine cocktail (4.4 mg/kg, IM). The expression and cellular localization of caveoln-1 were determined by Western blot and immunofluorescent study in rat urinary bladder. The study was approved by the Ethics Committee of the Chonnam National University Medical School.

\section{Cystometrogram}

Four weeks after the operation, rats ( $\mathrm{n}=10$ in each group) were anesthetized with $1.2 \mathrm{~g} / \mathrm{kg}$ urethane injected subcutaneously. A suprapubic midline incision was performed to expose the bladder, a transvesical catheter with a fire-flared tip (polyethylene catheter-50) was inserted into the dome of the bladder and secured with a ligature, and the abdomen was closed. The catheter was connected to a pressure transducer and syringe pump via a 3-way stopcock to record intravesical pressure and to infuse saline into the bladder. After the bladder was emptied, cystometry was performed with saline infused at a rate of $0.04 \mathrm{~mL} /$ min. The contraction pressure and contraction interval were recorded.

\section{Western Blot}

All minced tissues were homogenized in ice-cold isolation solution with a Tissumizer homogenizer (Tekmar, Cincinnati, OH, USA). Tissues were homogenized with five bursts of five strokes of a micro-sawtooth generator. Tissue homogenates $(n=10$ in each group, $50 \mu \mathrm{g}$ of protein) were separated by $12 \%$ sodium dodecyl sulfate-polyacrylamide gel electrophoresis and transferred to polyvinylidene difluoride membranes (Amersham Pharmacia Biotech, England, UK). The blots were then washed with Tris-Buffered Saline Tween-20 (TBS-T; $10 \mathrm{mM}$ Tris-HCl, $\mathrm{pH}$ 7.6, $150 \mathrm{mM} \mathrm{NaCl}, 0.05 \%$ Tween-20). The membrane was blocked with 5\% skimmed milk for 1 hour and incubated with the appropriate primary antibody. Monoclonal mouse antibodies for caveolin-1 (1:2,000; BD Biosciences Immunocytometry Systems, San Jose, CA, USA) and a polyclonal rabbit antibody against glyceraldehyde 3-phosphate dehydrogenase (GAPDH) (1:4,000; Cell Signaling Technology Inc., Danvers, MA, USA) were used. The membrane was then washed and caveolin-1 and GAPDH was detected with goat anti-mouse-immunoglobulin $\mathrm{G}$ (IgG) and goat anti-rabbit-IgG conjugated to horseradish peroxidase, respectively. Antibody incubations were performed in a $4^{\circ} \mathrm{C}$ incubator. The bands were visualized by enhanced chemiluminescence (Amersham Pharmacia Biotech, England, UK). GAPDH was used as an internal control. Densitometry analysis was performed with a Studio Star Scanner using NIH image ver. 1-57 (National. Institute of Health, Bethesda, MD, USA).

\section{Immunofluorescence Staining}

The tissue sections ( $\mathrm{n}=10$ in each group, 10 sections in each tissue) were rinsed in PBS, and then treated with normal chicken serum for 30 minutes to block nonspecific binding. After washing in PBS, the sections were incubated with antibodies for caveolin-1 (1:100; BD Biosciences Immunocytometry Systems, San Jose, CA, USA) in PBS for 12 to 14 hours at $4^{\circ} \mathrm{C}$. Immunoreactivity for caveolin-1 was detected using Alexa Fluor 594 chicken anti-mouse IgG $(\mathrm{H}+\mathrm{L})$ (Molecular Probes, Eugene, OR, USA). Tissues were mounted using mounting solution contained with 4'-6-diamidino-2-phenylindole. For a negative control, tissues were prepared in a similar manner, omitting caveolin-1 from the incubation solution. Tissues were examined with a LSM 510 (Carl Zeiss, Munchen-Hallbergmoos, Germany) confocal microscope with an excitation wavelength appro- 
priate for Alexa Fluor (405 nm) and Alexa Fluor (594 nm). Final images were constructed with LSM Image Examiner (Carl Zeiss) software.

\section{Statistical Analysis}

The results were expressed as the mean \pm standard deviation, whereas the data for cystometric parameters were expressed as the mean \pm standard error of mean. The Mann-Whitney test was used to test null hypothesis that there are no differences in the mean expression levels among the groups. Differences were considered significant at $\mathrm{P}<0.05$.

\section{RESULTS}

All animals survived for 3 days after intraperitoneal CYP injection. There was no significant difference in body weight and bladder weight between the groups.

\section{Effect of Cystitis on the Cystometric Parameters}

In cystometrograms, the contraction interval (minute) was significantly increased in the CYP-induced cystitis rats $(15.8 \pm 1.5)$ than in the control group $(6.3 \pm 0.5)(\mathrm{P}<0.05)$. And the contraction pressure $(\mathrm{mmHg})$ was significantly higher in the CYP-induced cystitis rats $(15.6 \pm 1.7)$ than in the control group (11.3 \pm $0.5)(\mathrm{P}<0.05)$ (Fig. 1).

\section{Effect of Cystitis on the Expression of Caveolin 1}

The expression of caveolin 1 was easily detected, expressed in areas of capillaries, venules and arteriols in subepithelial layer (Fig. 2). Immunofluorescence study revealed that, in terms of the cellular patterns of labeling, the expression of caveolin 1 in the cystitis group was decreased to that in the control groups (Fig. 2). Western blot analysis revealed bands at $22 \mathrm{kDa}$ corresponding to caveolin 1 protein (Fig. 3). Caveolin 1 protein was recognized in all groups. Caveolin 1 protein expression was significantly decreased in the CYP-induced cystitis rats $(\mathrm{P}<0.05)$ (Fig. 3).

\section{DISCUSSION}

This study has showed that the altered expression of caveolin 1 in the bladder following intraperitoneal injection of CYP in female rat urinary bladder. Three days after CYP injection, cystometric results exhibited a increased contraction interval and increased contraction pressure compared to control bladders. The immunohistochemical study showed that the localization of caveolin 1 in urinary bladder was detected in the capillaries, venules and arteriols in subepithlial layers. These results provide evidence that inflammatory condition of bladder induces bladder instability; caveolin 1 may play a role in the bladder dysfunction induced by cystitis in women. To our knowledge, this study is the first study showing the possible occurrence of signaling in the suburothelial microvasculature via caveolin 1 in bladder dysfunction from CYP induced cystitis rat model.

CYP-induced cystitis model is one of the most widely used models in lower urinary tract symptom and overactive bladder
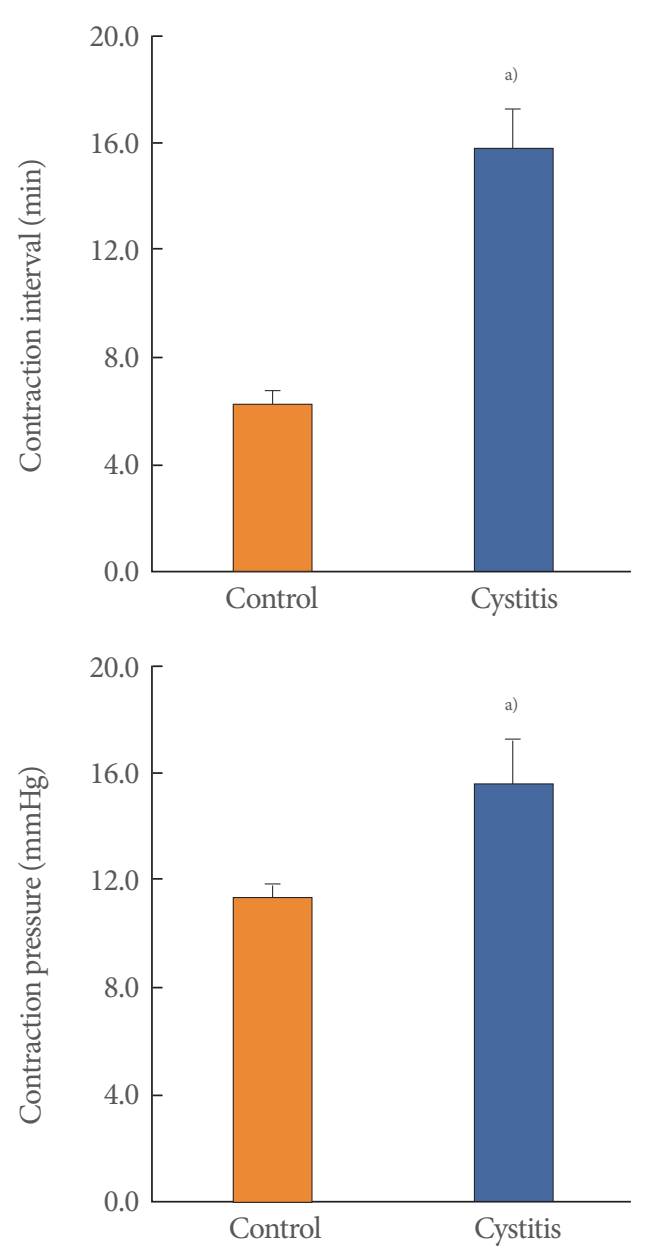

Fig. 1. Representative urodynamic profiles of control and cyclophosphamide-induced cystitis bladders. The contraction interval of the cystitis group increased significantly, compared with the control $\left({ }^{a} \mathrm{P}<0.05\right)$. Note the increased peak pressures on each voiding contraction in cystitis group compared to the control $\left.{ }^{(a)} \mathrm{P}<0.05\right)$. The lower panels denote the means \pm standard error of 10 experiments for each condition. ${ }^{\text {a) }} \mathrm{P}<0.05$ vs. control. 

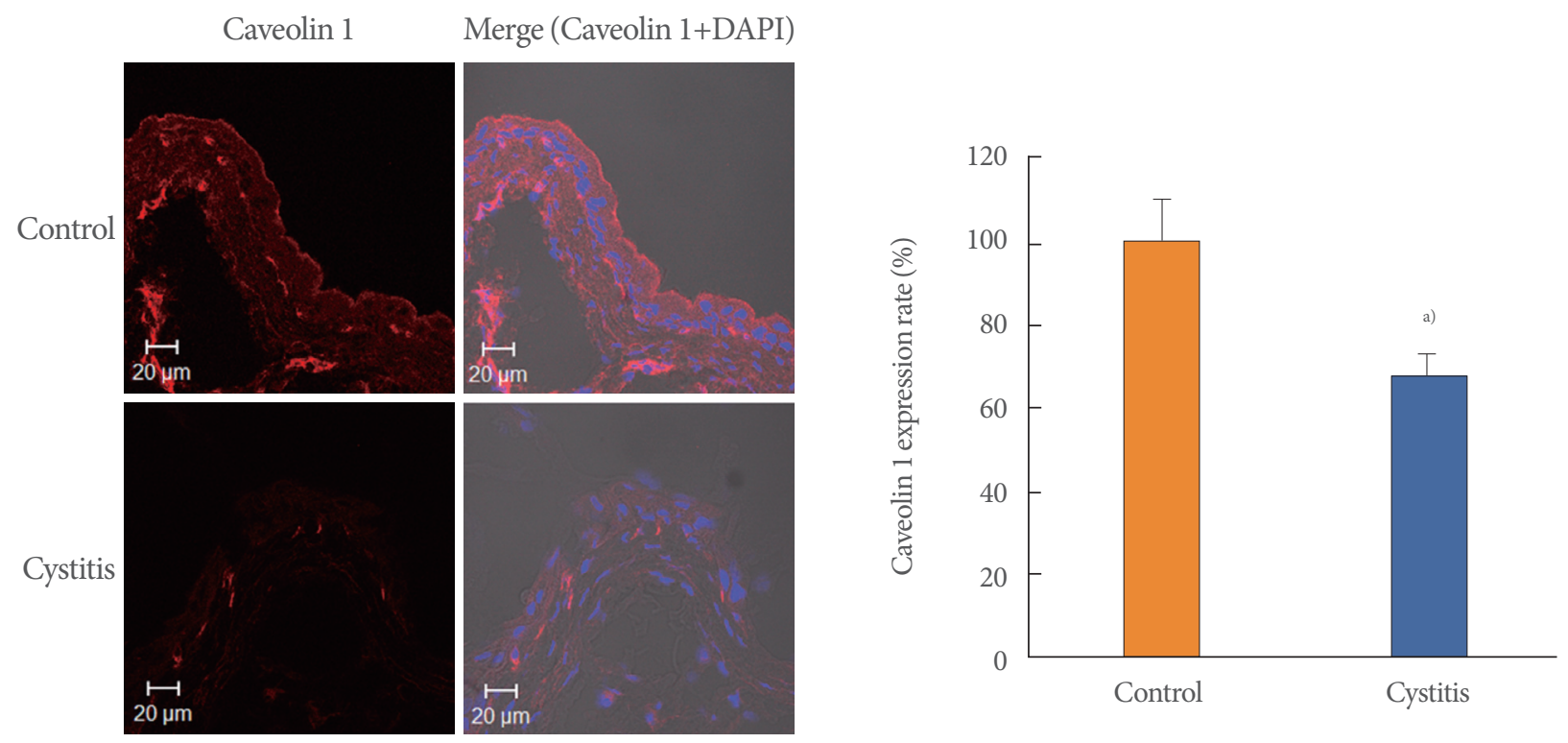

Fig. 2. Immunohistochemistry of caveolin 1 of rat urinary bladder tissue in control and cystitis group. Caveolin 1 was expressed throughout the capillaries, arteriols and venules. Immunofluorescence study revealed that, in terms of the cellular patterns of labeling, the expression of caveolin 1 in the cystitis group was decreased to that in the control groups. Horizontal scale bar at the bottom of each figure indicates the magnification power. The right panels denote the mean \pm standard deviation of 10 experiments for each condition determined by relative densitometry. DAPI, 4'-6-diamidino-2-phenylindole. ${ }^{\text {a) }} \mathrm{P}<0.05$.
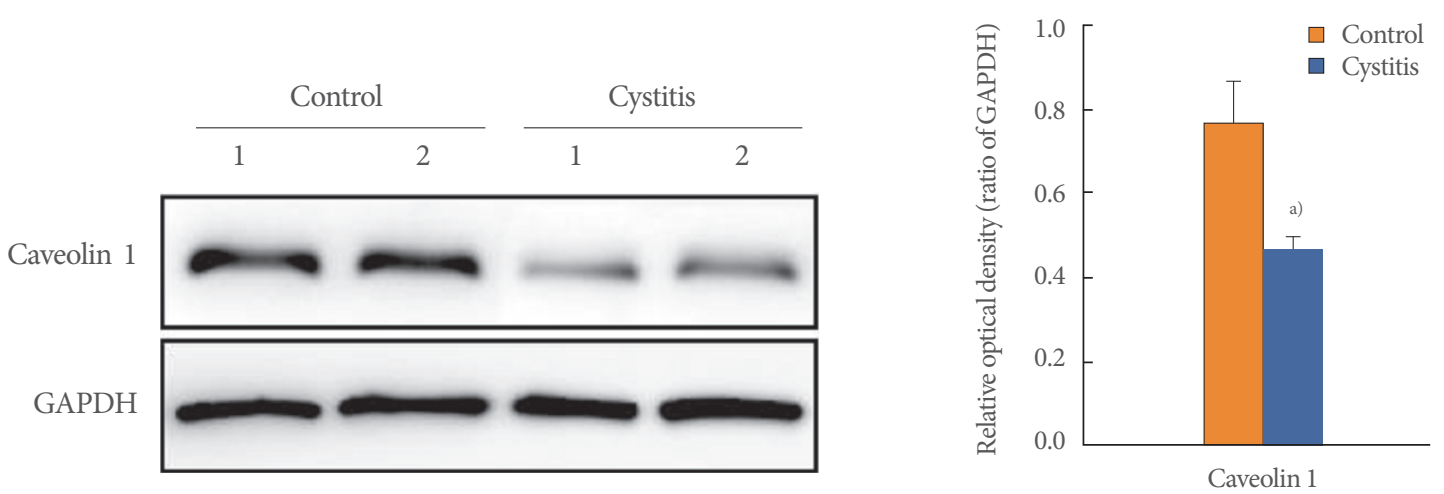

Fig. 3. Immunoblotting for caveolin 1 in urinary bladder tissue from animals of the control and cystitis groups. The anti-caveolin 1 antibodies recognize the $22 \mathrm{kDa}$ bands. Anti-glyceraldehyde 3-phosphate dehydrogenase (GAPDH) antibody recognizes the $42 \mathrm{kDa}$ band. The expression of caveolin 1 protein was significantly decreased in the cystitis group. The lower panels denote the mean \pm standard deviation of 10 experiments for each condition determined by densitometry relative to GAPDH. ${ }^{\text {a) }} \mathrm{P}<0.05$.

[7]. CYP induces chemical cystitis in animals and humans resulting in varying degrees of erosion of the mucosa, edema, hemorrhage, and leukocyte infiltration of the bladder wall $[8,9]$. The last few years has seen some significant advances in understanding of bladder sensory functions with the idea of crosstalk between the urothelium and the afferent nerves. Traditionally, the urothelium was considered to be a simple passive barrier between the urinary tract and urine. Recently, however, the urothelium is now understood to be active responsive organ capable of sensing a variety of signals from the urinary bladder [1]. In addition, a number of ion channels and receptors have also emerged as potential targets in the control of bladder function making it apparent that afferent mechanisms in the bladder may be more complex than previously thought [10].

More than 50 years ago, caveolae are 50 to $100 \mathrm{~nm}$ small subcellular structures that were originally described in capillary 
endothelial cell [11]. Caveolae are plasma membrane invaginations that play a role in cell signaling process involving such as endocytosis, and transcytosis in the endothelial and epithelial [12]. Caveolins are major constituents of caveolae [3]. Caveolin protein family consists of three members of caveolin 1, caveolin 2 and caveolin 3 that have a specific expression pattern to particular cell type. Among them, caveolin 1 is essential molecule for the induction of caveolae formation since they play a crucial role in maintaining the structure and shape of these membrane invaginations [13]. Signaling molecules are accumulated preferentially in these membrane invaginations such as G-proteins, endothelial nitric oxide synthase, epidermal growth factor receptors and some protein kinase-C isoenzymes that bind directly to the domain of caveolins [14].

There are number of studies indicating that caveolae may have an important role in bladder function in animal study. Caveolin 1 knockout mice showing lack of caveolae exhibited several urologic problem including decreased contractility on stimulation with carbachol in the bladder [4,5]. In hypertrophied bladder smooth muscle induced by bladder outlet obstruction, decreased caveolin was detected highlighting the dynamic nature of caveolae in urinary bladder [15]. Recently, Cristofaro et al. [16] reported that caveolin has a central role in regulation of G-protein coupled signaling pathways in bladder smooth muscle contraction. Our group previously reported that the hormonal influence on the caveolin 1 expression in urinary bladder showing that estrogen replacement increased expression of caveolin 1 protein in the rats urinary bladder [6].

However, there have been no studies to date investigating the expression of caveolin 1 in the suburothelial microvascular endothelial cells of urinary bladder or the changes in the functional activity of these proteins in response to cystitis condition. In the present study, the expression of caveolin-1 in the urinary bladder was significantly affected by CYP-induced cystitis group in association with the change of cystometric parameters. One of the possible reasons behind this influence on caveolin $1 \mathrm{ex}$ pression is believed to be the significance of the location of caveolin 1: the microvasculature is highly dependent on inflammatory condition and altered blood supply of the urinary bladder may also important step in regulating bladder function. Our results suggest that cystitis may lead to significant down-regulation of caveolin 1 expression in rat urinary bladder, providing presumptive evidence that caveolin 1 are involved in the affected lower urinary tract symptoms induced by cystitis, probably via modification of specific cellular transmission and signaling pathway. A limitation of our study is that the precise functional activity of caveolin 1 was not fully unveiled, although we did show the change in expression of caveolin 1 in the cystitis rat urinary bladder and possible role of relationship with caveolin 1 in the rat urinary bladder. Further studies are needed to investigate the expression and localization of all caveolin family members in the urinary bladder and to unveil functional role of these molecule in the underlying mechanisms of bladder pathophysiology.

In conclusion, this study showed that CYP-induced cystitis rats resulted in a significant decrease of the expression of caveolin 1 in rat urinary bladder. This may imply that caveolin 1 isoform might be involved in bladder signal activity and have a functional role in bladder dysfunction occured in association with inflammatory change of urinary bladder.

\section{CONFLICT OF INTEREST}

No potential conflict of interest relevant to this article was reported.

\section{ACKNOWLEDGEMENTS}

This research was supported by Basic Science Research Program through the National Research Foundation of Korea (NRF) funded by the Ministry of Education, Science and Technology (2009-0069443, 2010-1372, 2010-2296, 2011-2031, 2012-2139) and by a grant CRI11079-22, Chonnam National University Hospital Research Institute of Clinical Medicine. This work was supported by a research grant from the Research Institute of Medical Sciences, Chonnam National University (2011-CURIMSDR006).

\section{REFERENCES}

1. de Groat WC. The urothelium in overactive bladder: passive bystander or active participant? Urology 2004;64(6 Suppl 1):7-11.

2. Smart EJ, Ying Ys, Donzell WC, Anderson RG. A role for caveolin in transport of cholesterol from endoplasmic reticulum to plasma membrane. J Biol Chem 1996;271:29427-35.

3. Souto RP, Vallega G, Wharton J, Vinten J, Tranum-Jensen J, Pilch PF. Immunopurification and characterization of rat adipocyte caveolae suggest their dissociation from insulin signaling. J Biol Chem 2003;278:18321-9.

4. Lai HH, Boone TB, Yang G, Smith CP, Kiss S, Thompson TC, et al. 
Loss of caveolin-1 expression is associated with disruption of muscarinic cholinergic activities in the urinary bladder. Neurochem Int 2004;45:1185-93.

5. Lai HH, Boone TB, Thompson TC, Smith CP, Somogyi GT. Using caveolin-1 knockout mouse to study impaired detrusor contractility and disrupted muscarinic activity in the aging bladder. Urology 2007;69:407-11.

6. Kim SO, Song SH, Lee SC, Cho KA, Kim HS, Hwang IS, et al. Effects of estrogens on the expression of caveolin-1 in the urinary bladders of female rats. Int Neurourol J 2011;15:13-8.

7. Juszczak K, Gil K, Wyczolkowski M, Thor PJ. Functional, histological structure and mastocytes alterations in rat urinary bladders following acute and [corrected] chronic cyclophosphamide treatment. J Physiol Pharmacol 2010;61:477-82.

8. Wantuch C, Piesla M, Leventhal L. Pharmacological validation of a model of cystitis pain in the mouse. Neurosci Lett 2007;421:250-2.

9. Bjorling DE, Wang ZY, Bushman W. Models of inflammation of the lower urinary tract. Neurourol Urodyn 2011;30:673-82.
10. Yoshimura N. Lower urinary tract symptoms (LUTS) and bladder afferent activity. Neurourol Urodyn 2007;26(6 Suppl):908-13.

11. Palade GE. An electron microscope study of the mitochondrial structure. J Histochem Cytochem 1953;1:188-211.

12. Cohen AW, Hnasko R, Schubert W, Lisanti MP. Role of caveolae and caveolins in health and disease. Physiol Rev 2004;84:1341-79.

13. Li S, Couet J, Lisanti MP. Src tyrosine kinases, Galpha subunits, and H-Ras share a common membrane-anchored scaffolding protein, caveolin. Caveolin binding negatively regulates the auto-activation of Src tyrosine kinases. J Biol Chem 1996;271:29182-90.

14. Anderson RG. The caveolae membrane system. Annu Rev Biochem 1998;67:199-225.

15. Polyak E, Boopathi E, Mohanan S, Deng M, Zderic SA, Wein AJ, et al. Alterations in caveolin expression and ultrastructure after bladder smooth muscle hypertrophy. J Urol 2009;182:2497-503.

16. Cristofaro V, Peters CA, Yalla SV, Sullivan MP. Smooth muscle caveolae differentially regulate specific agonist induced bladder contractions. Neurourol Urodyn 2007;26:71-80. 